\title{
HIGH-RESOLUTION IMAGING OF THE DUST DISK AROUND 49 CETI
}

\author{
Z. WahHaj, ${ }^{1,2}$ D. W. Koerner, ${ }^{2}$ and A. I. Sargent ${ }^{3}$ \\ Received 2005 November 10; accepted 2007 January 11
}

\begin{abstract}
Subarcsecond scale Keck images of the young A1 V star, 49 Ceti, resolve emission at $\lambda=12.5$ and $17.9 \mu \mathrm{m}$ from a disk with long axis at position angle (P.A.) $125^{\circ} \pm 10^{\circ}$ and inclination $\phi=60^{\circ} \pm 15^{\circ}$. At $17.9 \mu \mathrm{m}$, the emission is brighter and more extended toward the northwest (NW) than the southeast (SE). Modeling of the mid-infrared images combined with flux densities from the literature indicate that the bulk of the mid-infrared emission comes from very small grains $(a \sim 0.1 \mu \mathrm{m})$ confined between 30 and $60 \mathrm{AU}$ from the star. This population of dust grains contributes negligibly to the significant excess observed in the spectral energy distribution. Most of the nonphotospheric energy is radiated at longer wavelengths by an outer disk of larger grains $(a \sim 15 \mu \mathrm{m})$, inner radius $\sim 60 \mathrm{AU}$, and outer radius $\sim 900$ AU. Global properties of the 49 Cet disk show more affinity with the $\beta$ Pic and HR 4796A disks than with other debris disks. This may be because they are all very young $(t<20 \mathrm{Myr})$, adding strength to the argument that they are transitional objects between Herbig Ae and "Vega-like" A stars with more tenuous circumstellar disks.
\end{abstract}

Subject headings: circumstellar matter — infrared: stars — planetary systems: formation planetary systems: protoplanetary disks — solar system: formation stars: individual (49 Ceti)

\section{INTRODUCTION}

Vega-excess stars were identified in observations with the Infrared Astronomical Satellite (IRAS) as having infrared excess emission attributed to circumstellar dust (see reviews by Backman \& Paresce 1993 and Zuckerman 2001). More than 100 Vegaexcess candidates have been identified by comparison of IRAS sources with stellar counterparts in optical catalogs since the early discovery of prototypes such as Vega and $\beta$ Pic (Backman \& Paresce 1993; Mannings \& Barlow 1998; Silverstone 2000). Estimated ages and circumstellar dust masses support the view that these objects are largely distinct from their pre-main-sequence counterparts, the T Tauri and Herbig Ae/Be stars. The latter have typical ages less than $10^{7} \mathrm{yr}$ and fractional infrared luminosities, $\tau=L_{\text {ir }} / L_{\text {bol }}$, of approximately 0.25 (Adams et al. 1987), while Vega-excess stars may be as old as $10^{9} \mathrm{yr}$ with values of $\tau$ typically in the range $10^{-4}$ to $10^{-7}$. Larger values of $\tau=10^{-3}$ are found for a few Vega-excess stars with much younger ages $\left(t \sim 10^{7} \mathrm{yr}\right)$ that often appear to be associated with young kinematic moving groups (Moór et al. 2006). Jura et al. (1998) noted three A-type stars in the Bright Star Catalogue with $\tau \sim 10^{-3}$ and interpreted them as representative of an early phase of debris disk evolution. $\beta$ Pic, HR 4796A, and 49 Cet have since been assigned stellar ages of $20 \mathrm{Myr}$ or less (Stauffer et al. 1995; Zuckerman et al. 1995; Zuckerman 2001) and may well be important as examples of the transition from viscous protostellar accretion disks to planetary systems. The existence of such a phase is supported by recent Spitzer studies of weak-line T Tauri stars in nearby clouds where a small sample of objects with values of $\tau$ in the high debris disk range was recently discovered by L. A. Cieza et al. (2007, in preparation).

High-resolution images can reveal features that signal the presence of gravitational interactions between unseen planets and orbiting dust grains. In recent years more than a dozen such disks have been resolved, revealing tantalizing features such as

\footnotetext{
1 Institute for Astronomy, University of Hawaii, Honolulu, HI 96814.

2 Northern Arizona University, Flagstaff, AZ 86011-6010.

3 Department of Astronomy, California Institute of Technology, Pasadena, CA 91125 .
}

ring arcs, clumps, disk warps, and other nonaxisymmetric signatures of potentially planetary importance (e.g., Holland et al. 1998; Koerner et al. 1998, 2001; Schneider et al. 1999, 2005; Heap et al. 2000; Wilner et al. 2002; Clampin et al. 2003; Wahhaj et al. 2003, 2005; Ardila et al. 2004; Liu 2004; Kalas et al. 2005, 2006). Simple gaps and rings are often apparent, although these may not be definitive signatures of unseen planets (Takeuchi \& Artymowicz 2001; Klahr \& Lin 2001). Some features, however, are as yet unaccounted for by any other means. Examples include stellar offset from the orbital center of an elliptical ring around Fomalhaut (Kalas et al. 2005). The evidence for a planet-disk connection is now sufficiently compelling that the Vega-excess phenomenon has been proposed as an indirect detection method for planetary systems (Zuckerman \& Song 2004).

Dynamically important disk features have been identified in high-resolution mid-infrared images for two out of the three young A-type stars mentioned by Jura et al. (1998). Keck studies of HR 4796A (Koerner et al. 1998; Telesco et al. 2000; Wahhaj et al. 2005) and $\beta$ Pic (Wahhaj et al. 2003; Weinberger et al. 2003; see also Gemini mid-infrared observations by Telesco et al. 2005) reveal gaps and rings with azimuthal asymmetries, inclination offsets, and radial changes in grain size and distribution. Here we present Keck mid-infrared imaging of 49 Cet, the third nearby A-type star identified by Jura et al. (1998) as having a young debris disk in an early transitional phase. 49 Ceti is an A1 V star with an age of $8 \pm 2$ Myr (Zuckerman et al. 1995; Thi et al. 2001 ) and a Hipparcos distance of $61 \pm 3$ pc. As discussed by Jayawardhana et al. (2001), mid-infrared radiation of 49 Cet (0.38 Jy in the IRAS $25 \mu \mathrm{m}$ band) is reduced in comparison to HR 4796A, posing an added challenge to imaging at mid-infrared wavelengths. Nevertheless, these authors report radiation from the circumstellar dust that is marginally resolved at $10.8 \mu \mathrm{m}$ with flux density $250 \pm 50 \mathrm{mJy}$. Substantial long-wavelength excess has also been detected at $\lambda=60 \mu \mathrm{m}$ by Sadakane $\&$ Nishida (1986) and at $\lambda=1.2 \mathrm{~mm}\left(F_{1.2 \mathrm{~mm}}=12.7 \mathrm{mJy}\right)$ by Bockelee-Morvan et al. (1994). Here we present Keck II images of 49 Cet at $\lambda=$ 12.5 and $17.9 \mu \mathrm{m}$ together with simultaneous modeling of both the images and spectral energy distribution. 


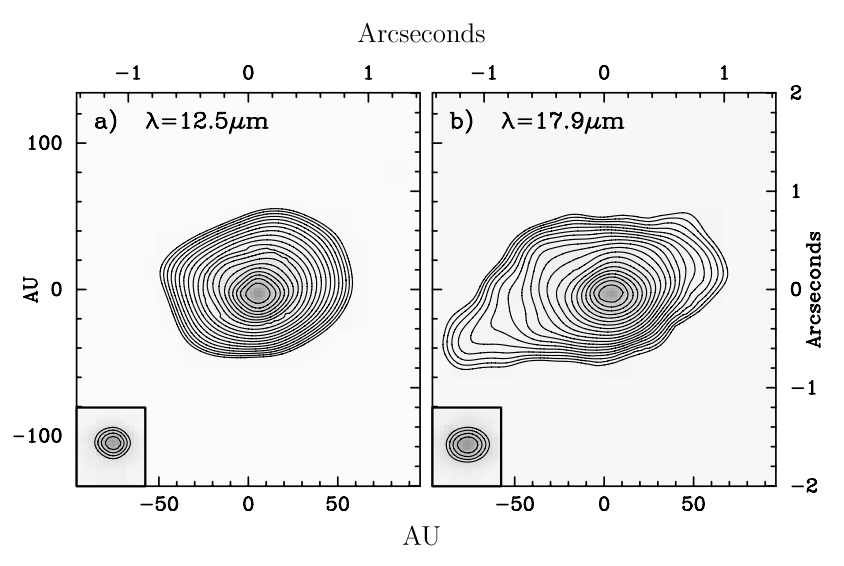

FIG. 1.-KECK MIRLIN contour maps of 49 Cet at $\lambda=12.5$ and $17.9 \mu \mathrm{m}$. North is up. Contour images of the PSF star, $\alpha$ Boo, are displayed in $(a)$ and (b) as insets with contour levels of $10 \%$, starting at the $60 \%$ level. The FWHM of the PSFs are $0.41^{\prime \prime}$ and $0.48^{\prime \prime}$ at 12.5 and $17.9 \mu$ m, respectively. (a) Emission at $12.5 \mu \mathrm{m}$. Lowest contour level is at the $2 \sigma$ level $\left(41 \mathrm{mJy} \mathrm{arcsec}^{-2}\right)$. Higher contour levels are at $2 \sigma\left(10^{0.068 n}\right)$ for the $n$th contour. (b) Emission at $17.9 \mu \mathrm{m}$ contoured as in (a). Lowest contour is at the $2 \sigma$ level $\left(26 \mathrm{mJy} a r c s e c^{-2}\right)$.

\section{OBSERVATIONS AND RESULTS}

The star 49 Cet was imaged with the Mid-Infrared Large-well Imager (MIRLIN; Ressler et al. 1994) at the f/40 bent-Cassegrain focus of the Keck II telescope on UT dates 1998 October 7 and 1999 July 28-31. At Keck, MIRLIN has a plate scale of $0.138^{\prime \prime}$ pixel $^{-1}$ and a $17.5^{\prime \prime}$ field of view. Filters centered on $\lambda=12.5$ and $17.9 \mu \mathrm{m}$ with widths of 1.16 and $2 \mu \mathrm{m}$ were used for the observations presented here. Initial sky subtraction was carried out by differencing images chopped $7^{\prime \prime}$ in an east-west direction at a frequency of $4 \mathrm{~Hz}$. The telescope was nodded the same distance in a northsouth direction after co-adding several hundred frames at each of the chop positions. The total integration times were 20 and 66 minutes at 12.5 and $17.9 \mu \mathrm{m}$, respectively. Residual background emission in the form of a striping pattern was removed by masking on-source emission and separately subtracting the median emission within each row and column of pixels. Disk emission in each of the double-differenced chop-and-nod frames was shifted and added to produce a final combined image. Observation and data reduction of standards $\alpha$ Boo, $\alpha$ Tau, $\chi$ Cet, and Vega proceeded in the same way for photometric calibration and served as a representation of the point-spread function (PSF). Images of 49 Cet and PSF stars from separate nights of observation were co-added after weighting by the signal-to-noise ratio of their peak flux. Seeing effects on low-level emission in the PSF stars were mostly evident at $12.5 \mu \mathrm{m}$ and were reduced for both the source and calibration images by smoothing with a circular hat function.

Final processed images of 49 Cet at angular resolutions of $0.41^{\prime \prime}$ and $0.48^{\prime \prime}$ at $\lambda=12.5$ and $17.9 \mu \mathrm{m}$, respectively, are shown in Figure 1 . The original $64 \times 64$ pixel $\left(8.8^{\prime \prime} \times 8.8^{\prime \prime}\right)$ images were finely gridded to $256 \times 256$ pixels and smoothed by convolution with a circular hat function of diameter equal to the full width at half-maximum (FWHM) of the PSF star $\alpha$ Boo. Images of $\alpha$ Boo, resampled and smoothed in the same manner, are shown in the insets in Figure 1. Images used later for model-fitting purposes were smoothed in the same way, but not resampled beforehand. In Figure 1 both images of 49 Cet are elongated along approximately the same position angle (P.A.). To characterize this effect more accurately, normalized strip cuts were obtained from the images by windowing the emission in rectangular apertures aligned with
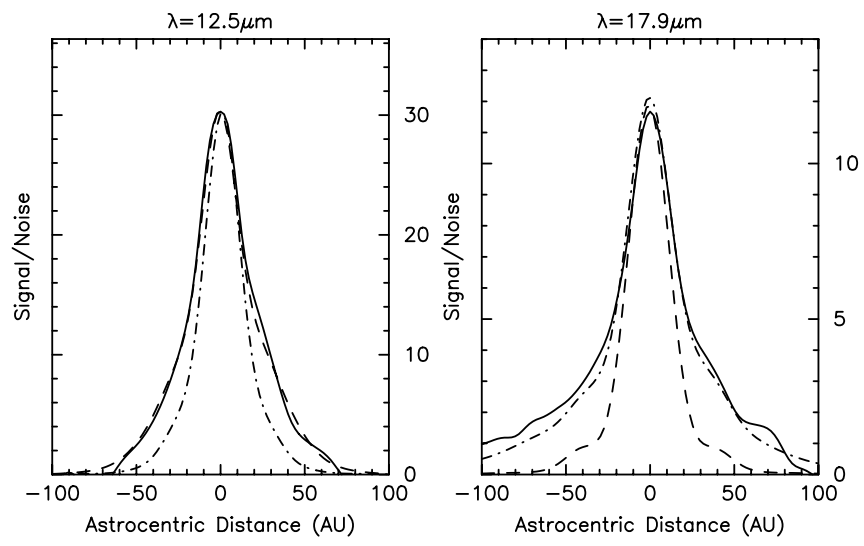

FIG. 2.-Flux density strip cuts of width equal to FWHMs of PSFs taken along the long axes of emission (P.A. $=125^{\circ}$ ) at 12.5 and $17.9 \mu \mathrm{m}$ seen in Fig. 1. Solid lines represent the 49 Cet emission profiles, and dot-dashed lines represent the profile for PSF star $\alpha$ Boo. Dashed lines are from best-fit models of emission described in $\S 3$ of the text.

the long axis of emission (P.A. $=125^{\circ}$ ) and of width corresponding to the PSF FWHM. The results, displayed in Figure 2, demonstrate that 49 Cet's emission profile is clearly resolved at both wavelengths.

Flux densities for 49 Cet were derived using aperture photometry of images of the calibrator stars. Calibrator flux densities were adopted from the Infrared Space Observatory (ISO) Calibration Web site ${ }^{4}$ as follows: $\alpha$ Boo $\left(F_{12.5}=478 \mathrm{Jy} ; F_{17.9}=232 \mathrm{Jy}\right), \alpha$ Tau $\left(F_{12.5}=423 \mathrm{Jy} ; F_{17.9}=205 \mathrm{Jy}\right), \chi \operatorname{Cet}\left(F_{12.5}=0.68 \mathrm{Jy}\right.$; $\left.F_{17.9}=0.33 \mathrm{Jy}\right)$, and Vega $\left(F_{12.5}=24.9 \mathrm{Jy} ; F_{17.9}=12.2 \mathrm{Jy}\right)$. The resulting flux densities for 49 Cet were $200 \pm 26$ and $186 \pm 25 \mathrm{mJy}$ at 12.5 and $17.9 \mu \mathrm{m}$. Although our $12.5 \mu \mathrm{m}$ value and the appreciably higher IRAS $12 \mu \mathrm{m}$ measurement (330 mJy) are at first sight inconsistent, the discrepancy is explained readily by the fact that the IRAS band is much broader $(6-18 \mu \mathrm{m})$ and thus includes more of the bright photospheric emission at shorter wavelengths. Peak brightnesses, the flux in the brightest pixel divided by the pixel area, were 231 and $583 \mathrm{mJy} \operatorname{arcsec}^{-2}$ at 12.5 and $17.9 \mu \mathrm{m}$, respectively. The corresponding rms background values were 41 and $26 \mathrm{mJy} \mathrm{arcsec}^{-2}$, respectively. The extent of the long axis enclosed by the $2 \sigma$ contours of the $12.5 \mu \mathrm{m}$ emission is $\sim 1.64^{\prime \prime}$ (100 AU). At $17.9 \mu \mathrm{m}$, elongation at the $2 \sigma$ level is $\sim 2.3^{\prime \prime}$ (140 AU) with the SE side clearly more extended than the NW side.

Available flux densities for 49 Cet at mid-infrared and longer wavelengths are listed in Table 1 . A $200 \mu \mathrm{m}$ flux density from ISOPHOT observations (Walker \& Heinrichsen 2000) was suspiciously low ( $0.32 \mathrm{Jy}$ ) compared to the value implied by an interpolation between the $100 \mu \mathrm{m} I R A S$ and $1.2 \mathrm{~mm}$ IRAM observations. We have therefore listed only the values obtained directly from the $I S O$ Data Archive $(1.1 \pm 0.5 \mathrm{Jy}$ at $170 \mu \mathrm{m}$ and $0.75 \pm 0.5 \mathrm{Jy}$ at $150 \mu \mathrm{m})$. Photometry in $U, B, V, J, H, K, L$, and $M$ bands from Sylvester et al. (1996) were used to constrain stellar photospheric properties as outlined below.

\section{MODELING}

We use a simple model of a flat optically thin disk with relatively few parameters (Backman et al. 1992; Koerner et al. 1998; Wahhaj et al. 2003, 2005) to derive the properties of the 49 Cet disk, since our images have limited spatial resolution, and

\footnotetext{
${ }^{4}$ See http://www.iso.vilspa.esa.es/users/expl_lib/ISO/wwwcal/.
} 
TABLE 1

Flux Densities for 49 CET

\begin{tabular}{|c|c|c|c|c|c|}
\hline $\begin{array}{c}\lambda_{\text {eff }} \\
(\mu \mathrm{m})\end{array}$ & $\begin{array}{c}\delta \lambda \\
(\mu \mathrm{m})\end{array}$ & $\begin{array}{l}\text { Flux Density } \\
\text { (Jy) }\end{array}$ & $\begin{array}{c}\text { Uncertainty } \\
(\mathrm{Jy})\end{array}$ & $\begin{array}{c}\text { Photosphere } \\
\text { (Jy) }\end{array}$ & Reference \\
\hline $10.8 \ldots \ldots \ldots \ldots \ldots \ldots \ldots \ldots \ldots \ldots \ldots \ldots \ldots$ & 5.3 & 0.25 & 0.05 & 0.191 & Jayawardhana et al. (2001) \\
\hline $12.5 \ldots \ldots \ldots \ldots \ldots \ldots$ & 1.2 & 0.2 & 0.026 & 0.143 & This work \\
\hline $17.9 \ldots \ldots \ldots \ldots \ldots \ldots \ldots \ldots \ldots \ldots \ldots \ldots$ & 2.0 & 0.186 & 0.025 & 0.069 & $\ldots$ \\
\hline $12.0 \ldots \ldots \ldots \ldots \ldots \ldots \ldots \ldots \ldots \ldots \ldots$ & 6.5 & 0.33 & 0.066 & 0.154 & $I R A S$ FSC \\
\hline $25.0 \ldots \ldots \ldots \ldots \ldots \ldots \ldots \ldots \ldots \ldots \ldots$ & 11.0 & 0.38 & 0.076 & 0.036 & $\ldots$ \\
\hline $60.0 \ldots \ldots \ldots \ldots \ldots \ldots \ldots$ & 40.0 & 2.0 & 0.4 & 0.006 & $\ldots$ \\
\hline 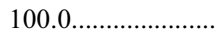 & 37.0 & 1.91 & 0.38 & 0.002 & $\ldots$ \\
\hline $150.0 \ldots \ldots \ldots \ldots \ldots \ldots \ldots \ldots \ldots \ldots$ & 40.0 & 0.75 & 0.50 & $\sim 0$ & ISO \\
\hline $170.0 \ldots \ldots \ldots \ldots \ldots \ldots$ & 50.0 & 1.1 & 0.50 & $\sim 0$ & $\ldots$ \\
\hline $800.0 \ldots \ldots \ldots \ldots \ldots \ldots . .$. & & $<0.036(3 \sigma)$ & & $\sim 0$ & Zuckerman \& Becklin (1993) \\
\hline $1200.0 \ldots \ldots \ldots \ldots \ldots$ & & 0.0127 & 0.0028 & $\sim 0$ & Bockelee-Morvan et al. (1994) (IRAM) \\
\hline
\end{tabular}

there are relatively few flux density measurements. We assume that thermal radiation from an annulus of width $d r$ at a distance $r$ from a star is given by

$$
f_{t}(r)=\sigma(r) \varepsilon_{\lambda} B\left[T_{p}(r), \lambda\right]\left(\frac{2 \pi r d r}{D^{2}}\right),
$$

where $\sigma(r)$ is the fractional surface density, $T_{p}(r)$ is the grain temperature, $B\left[T_{p}(r), \lambda\right]$ is the Planck function, and $D$ is the distance. For 49 Cet, $D=61 \mathrm{pc}$. Moderately absorbing dielectrics with an effective grain radius, $a$, have radiative efficiency $\varepsilon_{\lambda}=$ $1.5 a / \lambda$ for $\lambda>1.5 a$ and $\varepsilon_{\lambda}=1$ for $\lambda<1.5 a$ (Greenberg 1978). The grain temperature for efficient absorbers and inefficient emitters, $T_{p}(r)=432 a_{\mu \mathrm{m}}^{-0.2}\left(L_{*} / L_{\odot}\right)^{0.2}\left(r_{\mathrm{AU}}\right)^{-0.4} \mathrm{~K}$, can be calculated from radiative balance equations. For very small grains, $a<$ $0.05 \mu \mathrm{m}$, which are both inefficient absorbers and emitters, $T_{p}(r)=$ $636\left(L_{*} / L_{\odot}\right)^{2 / 11}\left(r_{\mathrm{AU}}\right)^{-4 / 11}\left(T_{*} / T_{\odot}\right)^{3 / 11} \mathrm{~K}$ (Backman \& Paresce 1993), where $L_{*}$ is the stellar luminosity, $18.4 L_{\odot}$ for 49 Cet. The two temperature laws converge at $a=0.05 \mu \mathrm{m}$, and varying the grain radius across this point does not result in a discontinuity in temperature. However, the latter law yields degenerate solutions between $\sigma(r)$ and $a$, and so only the product of the two quantities can be constrained. This degeneracy finds natural expression in the probability distributions generated by the Bayesian method, which we discuss later. The assumed power-law emissivities are admittedly crude approximations for the grain temperature behavior. However, the constraining power of the extant data is also limited. Thus, more realistic temperature laws are counterproductive, since they unnecessarily complicate the modeling method. The modeling constraints yielded on grain radii suggests a range of temperatures. It is better to work from these temperature ranges and investigate what kinds of dust grains they are consistent with, under more realistic modeling assumptions.

To describe the disk morphology and orientation we introduce parameters $R_{\text {in }}$, the inner radius; $\Delta R$, the width; $\gamma$, assuming radial density profile, $\sigma \sim r^{-\gamma} ; \phi$, the inclination of the disk to the line of sight $\left(90^{\circ}\right.$ is edge-on); and $\theta$, the P.A. of the long axis. We also include an asymmetry parameter $\beta$, the factor by which emission from the NW ansa exceeds that from the SE. We simulate the stellar photosphere by fitting a Kurucz (1993) model of a star with $T_{\text {eff }}=9250 \mathrm{~K}$ to the optical and infrared photometry of Sylvester et al. (1996), and use the mid-infrared and longer wavelengths estimates from Table 1 . The resulting photospheric flux density is added to the central pixel of the model image, and the final image is convolved with the appropriate PSF star to construct the simulated emission map. Models are varied over all pa- rameter space, and the probability of each model is calculated from $\chi^{2}$ following Wahhaj et al. (2005).

Initially, the two Keck MIRLIN images were treated separately in the model-fitting process to differentiate their separate roles in constraining disk parameter values. Orientation parameters such as $\phi$ and $\theta$ can be estimated only by fitting to these individual images. Most of the emission appears to arise in a small inner disk of very small $(a<1 \mu \mathrm{m})$ hot grains that contribute negligibly to the spectral energy distribution (SED). Both the temperature and the size of grains are mainly constrained by the relative brightness of the disk at $\lambda=12.5$ and $17.9 \mu \mathrm{m}$.

Our subsequent efforts to fit the SED and mid-infrared images simultaneously with a single disk model failed to reproduce the extensions in emission in the mid-infrared images. Unreduced $\chi^{2}$ values for the best fit were $\sim 45$, with cardinality of the data only 27 (see also in Wahhaj et al. 2005). Fits to the SED alone required a much larger disk than that mandated by the images to reproduce the long-wavelength flux densities and yielded only weak constraints on $R_{\text {in }}$ and grain radius $a$. We then invoked a two-part disk model comprising an inner disk of small grains to simulate the mid-infrared images and a large outer disk with a population of large dust grains to fit the SED. To introduce as few parameters as possible, the inner radius of the outer disk is set to begin at the outer edge of the inner disk. These two data sets are complimentary in that the $17.9 \mu \mathrm{m}$ image constrains the inner radius of the outer disk, while the SED limits the inner radius of the inner disk. This model effectively reproduces all the data and yields a $\chi^{2}$ of 28 . Most probable parameter values for the inner disk are $\phi=55^{\circ} \pm 20^{\circ}, \theta=120^{\circ} \pm 15^{\circ}, R_{\text {in }}=$ $30 \pm 10 \mathrm{AU}, \Delta R=30 \pm 20 \mathrm{AU}, a<1.0 \mu \mathrm{m}, \sigma=0.1-1.5 \times$ $10^{-3}$, and $\beta=0.5-2$, with no constraints on $\gamma$. The probability distribution for the grain radius, $a$, actually peaks around $0.1 \mu \mathrm{m}$. The distribution suggests no lower limit mostly because the temperature law becomes degenerate between grain radius and optical depth for $a<0.5 \mu \mathrm{m}$ as discussed earlier. Outer disk properties are $\phi=60^{\circ} \pm 15^{\circ}, \theta=125^{\circ} \pm 10^{\circ}, R_{\text {in }}=60 \pm 15 \mathrm{AU}, \Delta R=$ $900 \pm 400 \mathrm{AU}, a=15 \pm 10 \mu \mathrm{m}, \sigma=6.5 \pm 1.5 \times 10^{-4}, \beta=$ $1-10$, and $\gamma=0 \pm 0.5$. There exists an unrefereed publication of an $850 \mu \mathrm{m}$ JCMT SCUBA detection of 49 Cet (Song et al. 2004). At $8.2 \pm 1.9 \mathrm{mJy}$, this value is inconsistent with the $1.2 \mathrm{~mm}$ IRAM detection. We note that if the IRAM flux were ignored and instead the JCMT flux were used, the outer disk width would have to be $\sim 300 \mathrm{AU}$. The weak constraints on the disk asymmetry parameters can be explained by the fact that the average signal from the outer regions of the disk is roughly $3 \sigma$. Thus, any asymmetry seen in our images is statistically not very 


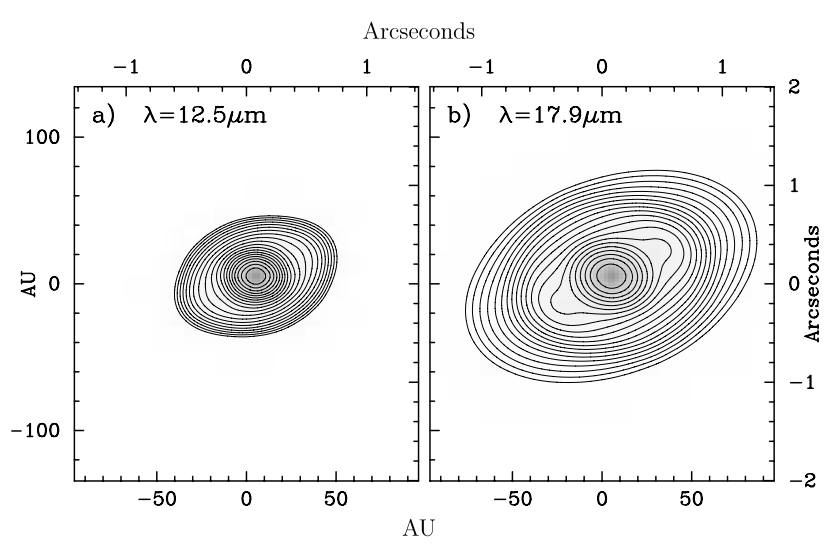

Fig. 3.-Simulated images at 12.5 and $17.9 \mu \mathrm{m}$ from the best-fit model. Contour levels are the same as in Fig. 1. For this model, $\phi=57^{\circ}, \theta=125^{\circ}, R_{\text {in }}=$ $35 \mathrm{AU}, \Delta R=10 \mathrm{AU}, a=0.1 \mu \mathrm{m}$, and $\sigma=1.3 \times 10^{-3}$ for the inner disk; and $\phi=60^{\circ}, \theta=125^{\circ}, R_{\text {in }}=45 \mathrm{AU}, \Delta R=900 \mathrm{AU}, a=23 \mu \mathrm{m}$, and $\sigma=5.4 \times$ $10^{-4}$ for the outer disk. Since only very weak constraints were found for the $\gamma$ - and $\beta$-parameters, they were set to the nominal values of 0 and 1 , respectively.

significant. The probability distributions for the inner/outer disk configurations are displayed in Figures 5 and 6.

Images simulated from the two-part disk model fit to Keck images and the SED for 49 Cet are displayed in Figure 3. The simulated SED is shown in Figure 4. We recognize that even this model may be an approximation to some more complicated structure that is not discernible from the current data set. Nevertheless,

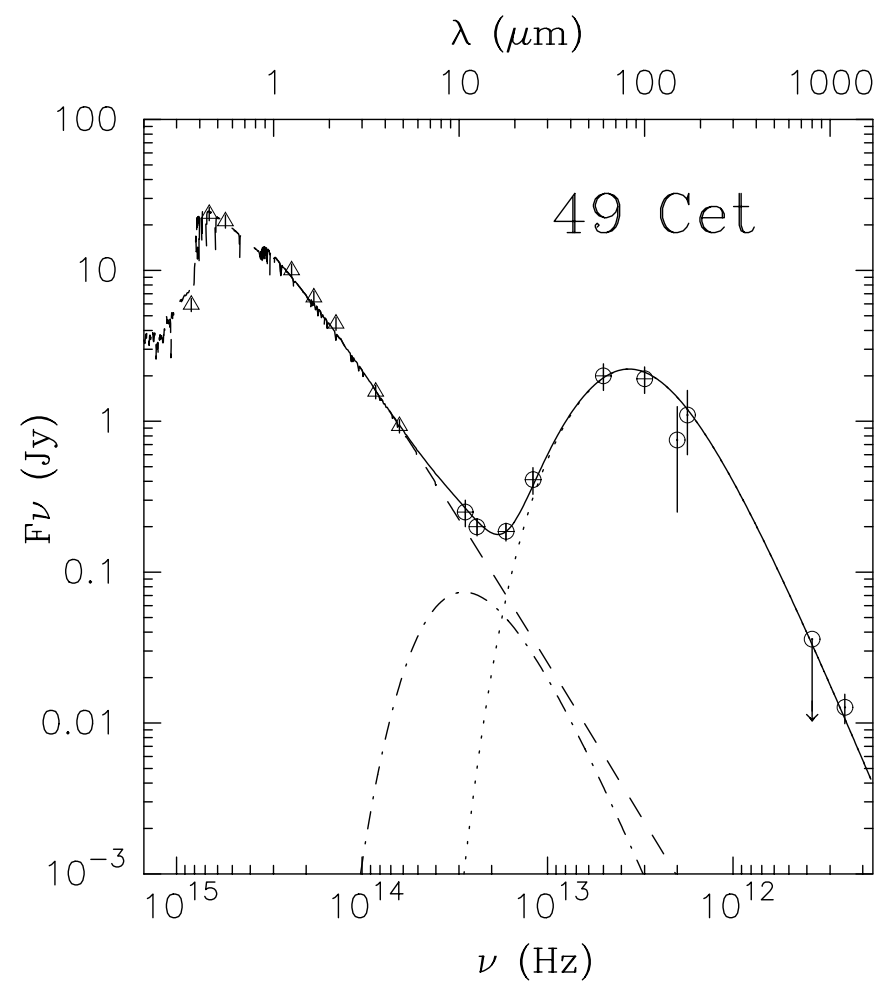

Fig. 4.-Flux densities of 49 Cet from Table 1 (circles) and UBVJHKL and $M$ photometry from Sylvester et al. (1996; triangles). The solid line represents the complete SED for the best-fit model shown in Fig. 3. The dashed line displays the contribution from the photosphere derived from a 1993 Kurucz model fit to the short-wavelength photometry. The dash-dotted line is the contribution from the inner disk suggested by the Keck mid-infrared images. The dotted line is contribution from the outer disk. a number of useful properties emerge: (1) the two-component disk has a uniform orientation, within uncertainties; (2) characteristic temperatures derived from a grain size-temperature relation (see Backman \& Paresce 1993) are $~ 360 \mathrm{~K}$ for inner dust grains and $\sim 95 \mathrm{~K}$ for the outer; (3) the total cross-sectional area of grains in the inner disk is $\sim 5 \mathrm{AU}^{2}$, and in the outer disk, $\sim 1650 \mathrm{AU}^{2}$; and (4) a grain density of $\rho=2500 \mathrm{~kg} \mathrm{~m}^{-3}$ (Jura 1998) leads to masses of $6.5 \times 10^{-6}$ and $0.32 M_{\oplus}$, respectively.

\section{DISCUSSION}

Mid-infrared images have also identified variations in radial structure in the $\beta$ Pic and HR 4796A disks. Structures with sharply varying density and grain size are apparent within the depleted inner region of $\beta$ Pic's disk (Lagage \& Pantin 1994; Backman et al. 1992; Wahhaj et al. 2003; Weinberger et al. 2003; Okamoto et al. 2004; Telesco et al. 2005). Likewise, a comparison of midinfrared and Hubble images of HR 4796A demonstrates that it is surrounded by a narrow ring of large grains embedded within a wider ring of smaller grains (Wahhaj et al. 2005), properties that are consistent with dispersal of an exo-Kuiper belt by radiation pressure. The analysis presented here suggests a similar morphology for 49 Cet. It is evident in Figure 5 that there is only a very small probability that the disk around 49 Cet extends all the way to the star. Indeed, it is most likely that the region interior to $20 \mathrm{AU}$ is strongly depleted of dust. The absence of silicate emission in Spitzer Space Telescope observations of 49 Cet suggests that the circumstellar dust must be cooler than $200 \mathrm{~K}$ (Kessler-Silacci et al. 2005). In combination with the constraints on grain size obtained here, this further strengthens the case for a large inner clearing of the disk.
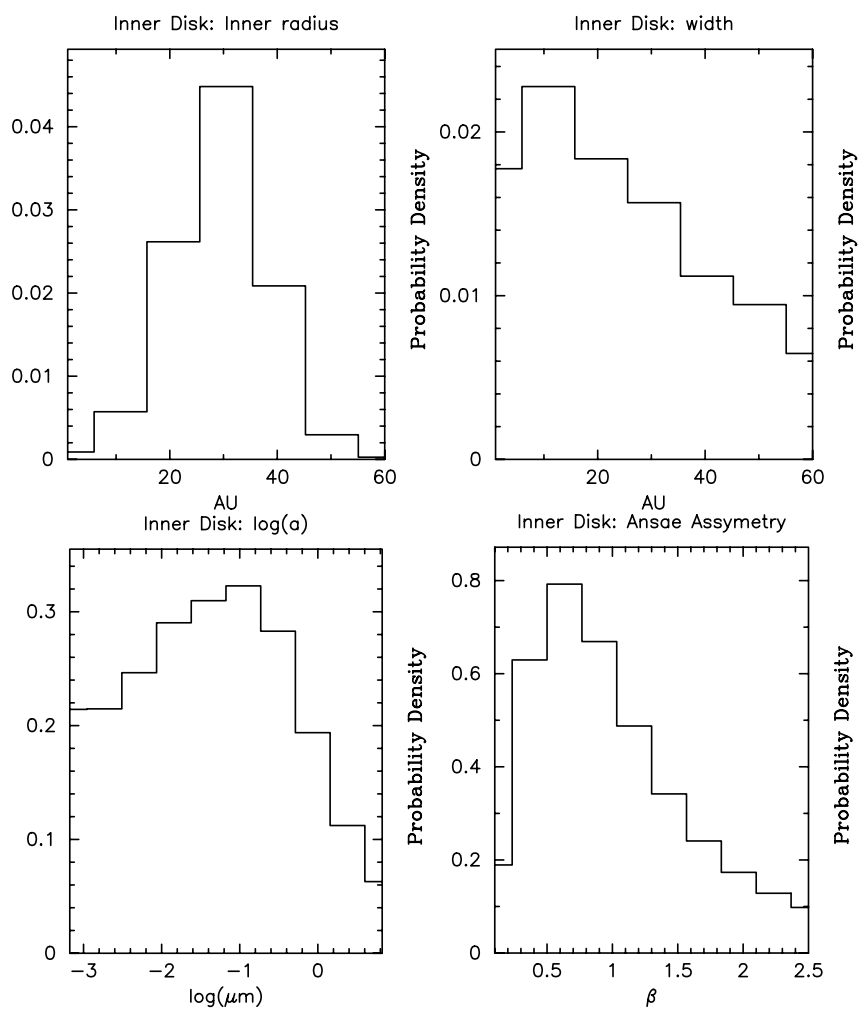

FIG. 5.-Probability distributions for selected inner disk model parameters. Most probable values correspond to peaks in the probability distributions. The uncertainties are estimated as the $66 \%$ confidence intervals (where our distributions are binned finely enough) or the shortest range of parameter values that encompasses $66 \%$ of the total probability. The most probable values and uncertainties for these parameters are detailed in the text. 

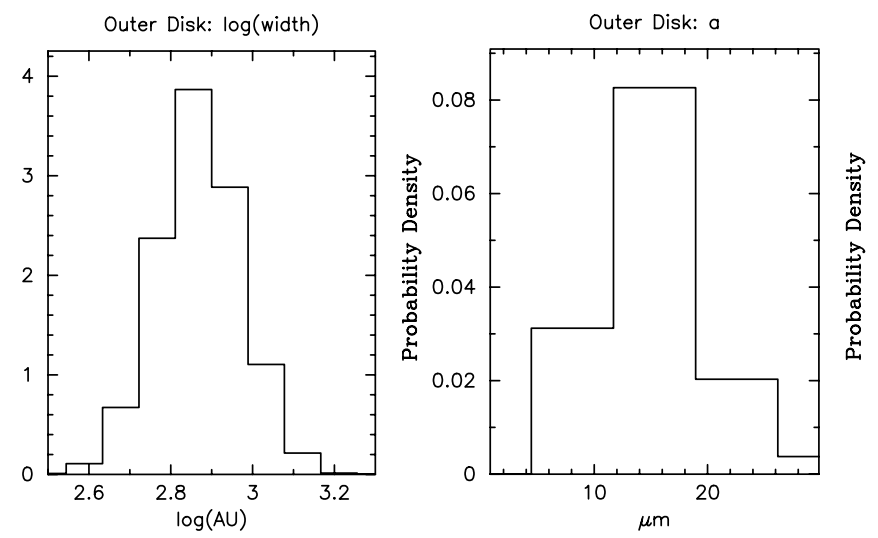

FIG. 6.- Probability distributions for selected outer disk model parameters.

The major contribution to the excess luminosity comes from a large-grained outer disk with an inner edge that can barely be discerned in the $17.9 \mu \mathrm{m}$ image. Both the 12.5 and $17.9 \mu \mathrm{m}$ images predominantly trace a region that is approximately 30-60 AU from the star and composed of very small dust grains, $a \sim 0.1 \mu \mathrm{m}$. How can dust grains as small as $0.1 \mu \mathrm{m}$ survive in the harsh radiation environment of 49 Cet? A conceivable explanation can be found in a paper by Artymowicz (1997). Grain-grain collisions result in a size distribution, $n \sim a^{-3.5}$ (Dohnanyi 1969). Grains smaller than $\sim 7 \mu \mathrm{m}$, similar to the blowout ( $\beta$-meteoroids) size for HR 4796A (Wahhaj et al. 2005), exit the system under radiation pressure. However, as they travel outward along the disk midplane, they undergo further collisions, producing a dust avalanche of even smaller grains. The resulting amplification in the dust production rate depends on the disk surface density and the width of the disk traversed. The smallest grains (called $\gamma$-meteoroids) will experience a relative pileup, since their outflow is braked more efficiently by residual gas (Thébault \& Augereau 2005). An approximate expression for dust production-amplification due to the avalanche phenomenon is $e^{N \sigma_{m}}$, where $N$ is the number of particles produced per collision and $\sigma_{m}$ is the fractional surface density experienced by a particle traveling along the disk midplane. Collision physics predicts values between $10^{2}$ and $10^{3}$ for $N$. Assuming that the disk thickness is $r / 10$, and the inner disk is $30 \mathrm{AU}$ wide, $\sigma_{m}=8 \times 10^{-3}$. Thus, the amplification factor ranges from 2 to 3000 . Detailed numerical simulations of the avalanche mechanism demonstrate that, in a debris disk with properties similar to 49 Cet, unbound grains from comet breakups can induce an amplification factor of 200 (Grigorieva et al. 2007). Obviously, too high a dust production rate predicts too short a disk lifetime. At the same time, rates consistent with the pileup of $\gamma$-meteoroids are accommodated by the range of predictions.

Another plausible mechanism for $\gamma$-meteoroid production is the sublimation of icy bodies analogous to comets or Kuiper Belt objects in our own solar system. Submicron refractory particles may be held together by volatile ices to produce large particles unaffected by radiation pressure. On sublimation, however, the smaller particles may be released and subsequently pushed outward. Indeed, transient redshifted lines in the $\beta$ Pic disks have been attributed to falling evaporating bodies (Beust et al. 1990, $1996,1998)$. While these events occur only for bodies that pass within $0.4 \mathrm{AU}$ of the star, sublimation of comets and asteroids can occur as far out as 4 AU (Flammer et al. 1998). Since the dust/ gas ratio in comets is close to 1 (Greenberg 1998), large amounts of evaporated fine dust particles might exist in the region of 49 Cet's inner disk. Given the wide-ranging grain sizes in optically thin circumstellar disks, from submicron-sized grains in $49 \mathrm{Cet}$ and HD 141569 (Marsh et al. 2002) to the $50 \mu \mathrm{m}$ grains in part of the disk around HR 4796A (Wahhaj et al. 2005), we conclude that multiple processes may control the evolution of dust grains both in size and radial location.

In summary, our analysis of 49 Cet, $\beta$ Pic, and HR 4796A demonstrates that their disks have more in common than just a large fractional luminosity. Morphological resemblances that set these objects apart from other "Vega-like" A stars, including $\alpha$ Psa and Vega itself may be due to their youth, 8-20 Myr (see discussion in Jura et al. 1993). They may in fact be in a transitional stage of evolution between that of viscous accretion disks around Herbig Ae stars (e.g., Mannings \& Sargent 1997; Mannings et al. 1997) and truly Vega-like stars with ages of order $100 \mathrm{Myr}$ or greater. As such, they are interesting candidates for constraints on the survival times of circumstellar gas. Marginal detection of molecular gas $\left(\sim 10^{-3} M_{\odot}\right)$ has been reported around 49 Cet (Thi et al. 2001) based on observations of the pure rotational transitions of $\mathrm{H}_{2}$. If circumstellar gas exists, it will be an important factor in the evolution of dust grains, since residual gas will shorten the lifetime of grains even as large as $\sim 50 \mu \mathrm{m}$ (Klahr $\&$ Lin 2001). Small amounts of CO were detected by Zuckerman et al. (1995) and Dent et al. (2005), but in regions close to the star $(<1.7 \mathrm{AU})$. Observations of $\mathrm{CO}(3 \rightarrow 2)$ at $850 \mu \mathrm{m}$ by Coulson et al. (1998) suggest a very low upper limit on the gas mass in the disk $<6 \times 10^{-8} M_{\odot}$. CO is easily frozen out onto grains in the colder parts of the disk (Aikawa et al. 1997).

A better understanding of both the abundance of molecular gas and the morphology of cold dust in the outer disk await study at longer wavelengths with higher resolution and sensitivity, such as will be available to the next generation of millimeter wave arrays.

Observations presented here were obtained at the W. M. Keck Observatory (WMKO), which is operated as a scientific partnership among the California Institute of Technology, the University of California, and the National Aeronautics and Space Administration. The Observatory was made possible by the generous financial support of the W. M. Keck Foundation. A great debt is due, also, to Robert Goodrich and the WMKO summit staff for their many hours of assistance in adapting MIRLIN to the Keck II visitor instrument port. The authors wish also to recognize and acknowledge the very significant cultural role and reverence that the summit of Mauna Kea has always had within the indigenous Hawaiian community. We are most fortunate to have the opportunity to conduct observations from this mountain.

\section{REFERENCES}

Adams, F. C., Lada, C. J., \& Shu, F. H. 1987, ApJ, 312, 788

Aikawa, Y., Umebahashi, T., Nakano, T., \& Miyama, S. M. 1997, ApJ, 486, L51

Ardila, D. R., et al. 2004, ApJ, 617, L147

Artymowicz, P. 1997, Annu. Rev. Earth Planet. Sci., 25, 175
Backman, D. E., Gillett, F. C., \& Witteborn, F. C. 1992, ApJ, 385, 670

Backman, D. E., \& Paresce, F. 1993, in Protostars and Planets III, ed. E. H. Levy \& J. I. Lunine (Tucson: Univ. Arizona Press), 1253

Beust, H., Lagrange, A.-M., Crawford, I. A., Goudard, C., Spyromilio, J., \& Vidal-Madjar, A. 1998, A\&A, 338, 1015 
Beust, H., Lagrange, A.-M., Plazy, F., \& Mouillet, D. 1996, A\&A, 310, 181 Beust, H., Vidal-Madjar, A., Ferlet, R., \& Lagrange-Henri, A. M. 1990, A\&A, 236, 202

Bockelée-Morvan, D., André, P., Colom, P., Colas, F., Crovisier, J., Despois, D., \& Jorda, L. 1994, in Circumstellar Dust Disks and Planet Formation, ed. R. Ferlet \& A. Vidal-Madjar (Gif-sur-Yvette: Ed. Frontières), 341

Clampin, M., et al. 2003, AJ, 126, 385

Coulson, I. M., Walther, D. M., \& Dent, W. R. F. 1998, MNRAS, 296, 934

Dent, W. R. F., Greaves, J. S., \& Coulson, I. M. 2005, MNRAS, 359, 663

Dohnanyi, J. W. 1969, J. Geophys. Res., 74, 2531

Flammer, K. R., Mendis, D. A., \& Houpis, H. L. F. 1998, ApJ, 494, 822

Greenberg, J. M. 1978, in Infrared Astronomy, ed. G. Setti \& G. G. Fazio (NATO ASI Ser. C38; Dordrecht: Reidel), 51

. 1998, A\&A, 330, 375

Grigorieva, A., Artymowicz, P., \& Thebault, P. 2007, A\&A, 461, 537

Heap, S. R., Lindler, D. J., Lanz, T. M., Cornett, R. H., Hubeny, I., Maran, S. P., \& Woodgate, B. 2000, ApJ, 539, 435

Holland, W. S., et al. 1998, Nature, 392, 788

Jayawardhana, R., Fisher, R. S., Telesco, C. M., Piña, R. K., Barrado y Navascués, D., Hartmann, L. W., \& Fazio, G. G. 2001, AJ, 122, 2047

Jura, M., Malkan, M., White, R., Telesco, C., Pena, R., \& Fisher, R. S. 1998, ApJ, 505, 897

Jura, M., Zuckerman, B., Becklin, E. E., \& Smith, R. C. 1993, ApJ, 418, L37

Kalas, P., Graham, J. R., Clampin, M. C., \& Fitzgerald, M. P. 2006, ApJ, 637, L57

Kalas, P., et al. 2005, in Nearby Resolved Debris Disks Conference, ed. I. Kamp \& M. Meixner (Baltimore: STScI), 13

Kessler-Silacci, J. E., Hillenbrand, L. A., Blake, G. A., \& Meyer, M. R. 2005, ApJ, 622, 404

Klahr, H. H., \& Lin, D. N. C. 2001, ApJ, 554, 1095

Koerner, D. W., Ressler, M. E., Werner, M. W., \& Backman, D. E. 1998, ApJ, 503, L83

Koerner, D. W., Sargent, A. I., \& Ostroff, N. A. 2001, ApJ, 560, L181

Kurucz, R. L. 1993, CD-ROM 13, ATLAS9 Stellar Atmosphere Programs and $2 \mathrm{~km} / \mathrm{s}$ Grid (Cambridge: SAO)

Lagage, P. O., \& Pantin, E. 1994, Nature, 369, 628

Liu, M. C. 2004, Science, 305, 1442
Mannings, V., \& Barlow, M. J. 1998, ApJ, 497, 330

Mannings, V., Koerner, D. W., \& Sargent, A. I. 1997, Nature, 388, 555

Mannings, V., \& Sargent, A. I. 1997, ApJ, 490, 792

Marsh, K. A., Silverstone, M. D., Becklin, E. E., Koerner, D. W., Werner, M. W., Weinberger, A. J., \& Ressler, M. E. 2002, ApJ, 573, 425

Moór, A., Ảbrahám, P., Derekas, A., Kiss, C., Kiss, L. L., Apai, D., Grady, C., \& Henning, T. 2006, ApJ, 644, 525

Okamoto, Y. K., et al. 2004, Nature, 431, 660

Ressler, M. E., Werner, M. W., Van Cleve, J., \& Chou, H. A. 1994, Exp. Astron., 3,277

Sadakane, K., \& Nishida, M. 1986, PASP, 98, 685

Schneider, G., Silverstone, M. D., \& Hines, D. C. 2005, ApJ, 629, L117

Schneider, G., et al. 1999, ApJ, 513, L127

Silverstone, M. D. 2000, Ph.D. thesis, Univ. California, Los Angeles

Song, I., Sandell, G., \& Friberg, P. 2004, in ASP Conf. Ser. 324, Debris Disks and the Formation of Planets: A Symposium in Memory of Fred Gillett, ed. L. Caroff et al. (San Francisco: ASP), 250

Stauffer, J. R., Hartmann, L. W., \& Barrado y Navascues, D. 1995, ApJ, 454, 910 Sylvester, R. J., Skinner, C. J., Barlow, M. J., \& Mannings, V. 1996, MNRAS, 279, 915

Takeuchi, T., \& Artymowicz, P. 2001, ApJ, 557, 990

Telesco, C. M., et al. 2000, ApJ, 530, 329 2005, Nature, 433, 133

Thébault, P., \& Augereau, J.-C. 2005, A\&A, 437, 14

Thi, W. F., et al. 2001, ApJ, 561, 1074

Wahhaj, Z., Koerner, D. W., Backman, D. E., Werner, M. W., Serabyn, E., Ressler, M. E., \& Lis, D. C. 2005, ApJ, 618, 385

Wahhaj, Z., Koerner, D. W., Ressler, M. E., Werner, M. W., Backman, D. E., \& Sargent, A. I. 2003, ApJ, 584, L27

Walker, H. J., \& Heinrichsen, I. 2000, Icarus, 143, 147

Weinberger, A. J., Becklin, E. E., \& Zuckerman, B. 2003, ApJ, 584, L33

Wilner, D. J., Holman, M. J., Kuchner, M. J., \& Ho, P. T. P. 2002, ApJ, 569, L115 Zuckerman, B. 2001, ARA\&A, 39, 549

Zuckerman, B., \& Becklin, E. E. 1993, ApJ, 406, L25

Zuckerman, B., Forveille, T., \& Kastner, J. H. 1995, Nature, 373, 494

Zuckerman, B., \& Song, I. 2004, ApJ, 603, 738 